Rechtsmedizin 2021 · 31:501-508

https://doi.org/10.1007/s00194-021-00468-7

Angenommen: 23. Januar 2021

Online publiziert: 22. Februar 2021

(c) Der/die Autor(en) 2021

\section{Einleitung}

$\mathrm{Zu}$ den Aufgaben in der rechtsmedizinischen Praxis gehören zunehmend forensisch-medizinische Untersuchungen von Lebenden. In den letzten Jahren sind zahlreiche Studien über körperliche Untersuchungen von Gewaltopfern in der Fachliteratur erschienen [1-6]. Dabei werden von den Ärzten neben den im Zusammenhang mit Straftaten geschädigten Personen aber ebenso Tatverdächtige untersucht. Im Gegensatz zu Studien über Gewaltopfer findet sich in der Fachliteratur ein erhebliches Defizit an Ergebnissen über systematische rechtsmedizinische Untersuchungen von Tatverdächtigen [7, 8]. Mit der vorliegenden Studie im Rahmen einer Dissertation sollen Daten einer retrospektiven Analyse von forensisch-medizinischen Untersuchungen von Tatverdächtigen aus dem Einzugsbereich des Rostocker Instituts für Rechtsmedizin vorgestellt werden.

\section{Material und Methode}

Von den Ärzten des Instituts für Rechtsmedizin der Universitätsmedizin Rostock wurden im Zeitraum von 2006 bis 2018 bevorzugt im Auftrag der Staatsanwaltschaften Rostock und Schwerin insgesamt 1232 forensisch-medizinische Untersuchungen von Personen durchgeführt. Von diesen Gutachten waren

F. Zack · S. Bernhardt · C. Arnold · A. Büttner

Institut für Rechtsmedizin, Universitätsmedizin Rostock, Rostock, Deutschland

\title{
Forensisch-medizinische Untersuchungen von Tatverdächtigen. Eine Analyse von 270 Gutachten aus den Jahren 2006 bis 2018
}

insgesamt $270(21,9 \%)$ Untersuchungen von Tatverdächtigen.

Diese Gutachten wurden nach den Kategorien

1. allgemeine Ergebnisse,

2. Tatgeschehen,

3. Tatverdächtige und

4. Relevanz der Gutachten für die

Ermittlungs-/Strafverfahren

ausgewertet.

Neben den schriftlichen Gutachten, einschließlich dem angegebenen polizeilichen/staatsanwaltschaftlichen Ermittlungsstand und vorhandener Fotodokumentation, wurden auch, wenn fallbezogen vorliegend, rechtsmedizinische Leichenschauen und Obduktionen berücksichtigt. In einzelnen Fällen, in denen die Untersucher als Gutachter bei Gerichtsverhandlungen geladen waren, wurden auch der Inhalt und Ausgang des Strafverfahrens, einschließlich Gerichtsurteile, einbezogen. Die Einteilung der Gutachten hinsichtlich der Relevanz für die Ermittlungs-/Strafverfahren erfolgte im Nachhinein aus der Zusammenschau aller vorliegenden Ergebnisse und Informationen aus rechtsmedizinischer Sicht.

Die rechtliche Qualifikation der Tat wurde aus pragmatischen Gründen ebenfalls aus forensisch-medizinischer Sicht vorgenommen, da die juristische Sicht in zahlreichen Fällen zum Zeitpunkt der Gutachtenerstellung unbekannt geblieben war und es aus beruflicher Erfahrung im Laufe eines Strafverfahrens nicht selten zu Änderungen der rechtlichen Qualifikation aus juristischer Sicht (Polizei - Staatsanwaltschaft - Gericht) kommt.

\section{Ergebnisse}

\section{Allgemeine Ergebnisse}

Die Anzahl der Gutachten über Tatverdächtige schwankte im Untersuchungszeitraum beträchtlich. Es war weder ein eindeutiger Trend zur Zunahme noch zur Abnahme derartiger Untersuchungen bis $2018 \mathrm{zu}$ verzeichnen (• Abb. 1).

Von den 270 Gutachten waren nur in 57 Fällen (21,1 \%) vom Auftraggeber zum Zeitpunkt der Auftragserteilung Fragen gestellt worden. Diese betrafen u. a. die Sitzposition des Tatverdächtigen in einem Pkw nach einem Verkehrsunfall mit mehr als einem Insassen, die Differenzierung zwischen Selbst- und Fremdbeibringung oder die Fragen nach der Ursache der festgestellten Verletzungen.

Eine zeitgleich zur Untersuchung erfolgte Spurensicherung durch Rechtsmediziner wurde in 91 Fällen $(33,7 \%)$ durchgeführt. Angaben über Spurensicherungen durch andere Personen (Mitarbeiter der Polizei, Frauenklinik etc.) waren den Gutachten nicht zu entnehmen.

Mit 164 (60,7\%) Untersuchungen fand die Mehrheit innerhalb der ersten $24 \mathrm{~h}$ nach dem angegebenen Tatgeschehen statt (• Abb. 2). Bei den aufgesuchten 


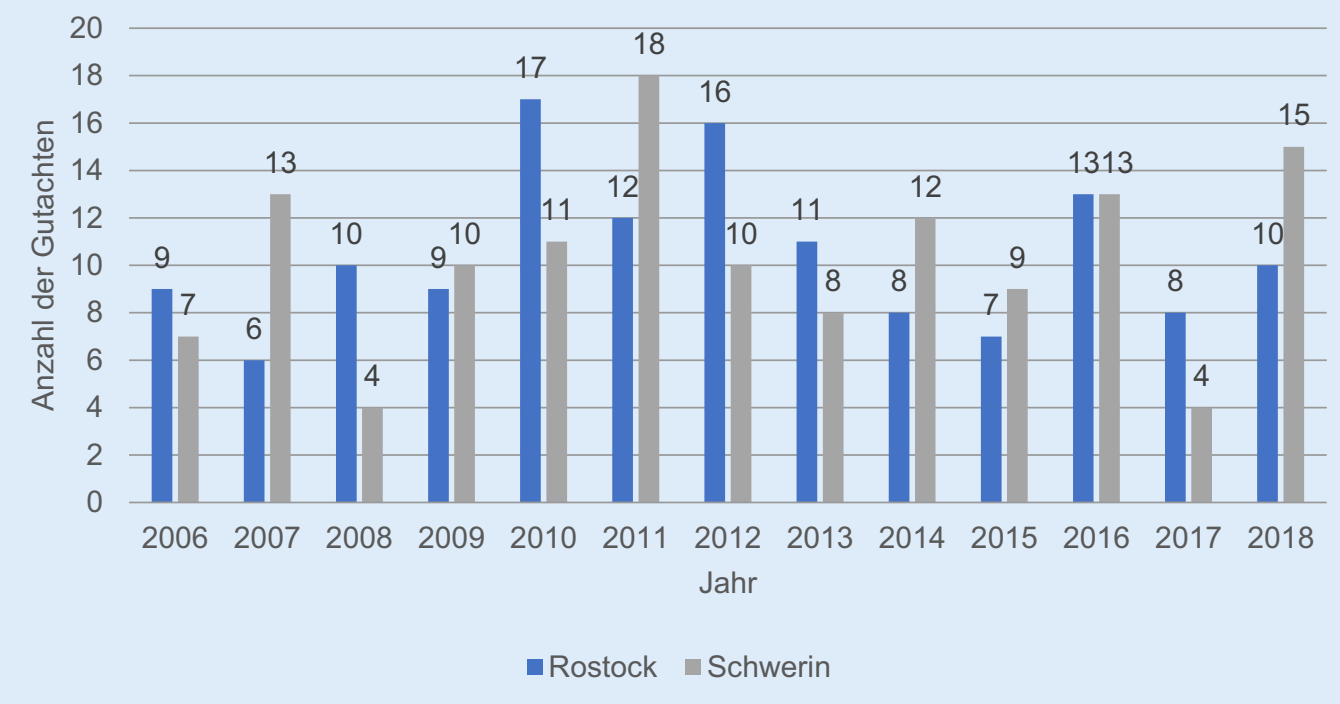

Abb. $1 \varangle$ Häufigkeiten der körperlichen Untersuchungen von Tatverdächtigen der Jahre 2006 bis 2018

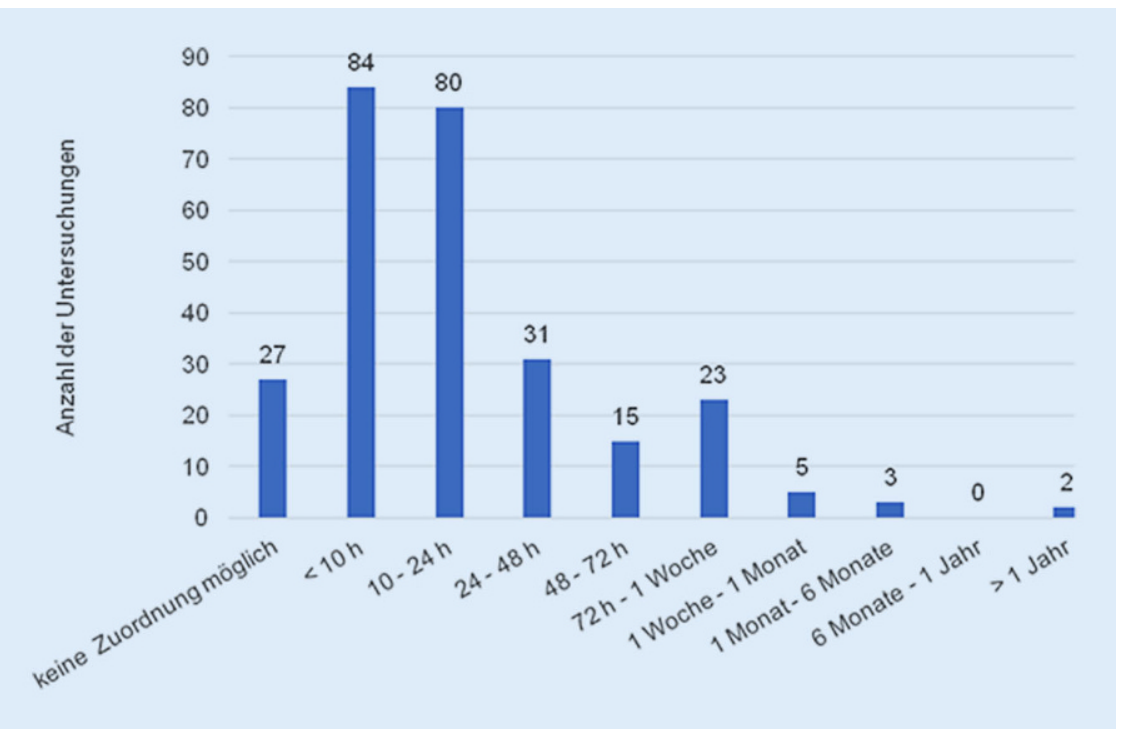

Abb. 2 \ Absolute Häufigkeit der Untersuchungen nach Zeitspanne zwischen Tat und Begutachtung

Untersuchungsorten dominierten mit 209 Fällen $(75,2 \%)$ die Polizeireviere (• Abb. 3).

$\mathrm{Zu}$ den 270 Gutachten über Tatverdächtige gab es 209 (77,4\%) korrespondierende Opferuntersuchungen. Von diesem Teilkollektiv der 209 Fälle mit Täter-Opfer-Begutachtungen wurden in 193 Fällen (92,3\%) Tatverdächtige und Opfer von demselben Gutachter untersucht. Eine explizite Anforderung von zwei verschiedenen Gutachtern zur Vermeidung von Spurenübertragungen gab es in keinem Fall.

\section{Tatgeschehen}

Die untersuchten 270 Tatverdächtigen wurden beschuldigt, insgesamt 222 Taten begangen zu haben. Von den 270 Personen waren 194 alleinhandelnde Beschuldigte $(71,9 \%)$.

Bei den Tatorten war auffällig, dass von den 222 Straftaten in zahlreichen Fällen $(n=73=32,8 \%)$ in den Gutachten keine Angaben über den genauen Tatort dokumentiert waren. Unter den verbliebenen 149 Fällen dominierte die Öffentlichkeit mit 39 (26,2\%) Angaben. Die Wohnung des Tatverdächtigen $(n=16=10,7 \%)$ war wesentlich seltener als die Wohnung des Opfers $(n=29=19,5 \%)$ der Tatort (• Abb. 4). Die vorgeworfenen Straftaten waren am häufigsten Körperverletzungen $(n=88=$ $30,7 \%$ ), gefolgt von vorsätzlichen Tötungen $(n=63=22,0 \%)$ und Sexualdelikten $(n=49=17,1 \%$; D Abb. 5$)$.

\section{Tatverdächtige}

Von den begutachteten Personen waren $236(87,4 \%)$ männlichen und $34(12,6 \%)$ weiblichen Geschlechts. Die meisten Tatverdächtigen $(n=175=65,5 \%)$ waren in einem Lebensalter von 18 bis 40 Jahren. Bei 39 Tatverdächtigen war dem Arzt die Nationalität am Tag der Untersuchung nicht bekannt. Von den verbliebenen 231 untersuchten Personen hatten $212(91,8)$ die deutsche Staatsbürgerschaft.

In den Gutachten wurden insgesamt 544 Verletzungen durch stumpfe, 110 durch halbscharfe und 68 durch scharfe Gewalteinwirkungen festgestellt. Unter 12 andersartigen Befunden fanden sich Schuss- und Bissverletzungen, Verbrennungen, Punktionsmale und Auftragungen von Kosmetika.

\section{Relevanz der Gutachten für die Ermittlungs-/Strafverfahren}

Im Hinblick auf die Aussage der rechtsmedizinischen Untersuchungsergebnisse in Bezug auf den vorgeworfenen Straftatbestand wurden im Nachhinein 126 
Rechtsmedizin 2021 ·31:501-508 https://doi.org/10.1007/s00194-021-00468-7

(c) Der/die Autor(en) 2021

\section{F. Zack · S. Bernhardt · C. Arnold · A. Büttner}

\section{Forensisch-medizinische Untersuchungen von Tatverdächtigen. Eine Analyse von 270 Gutachten aus} den Jahren 2006 bis 2018

\section{Zusammenfassung}

Hintergrund. Zu den Aufgaben in der rechtsmedizinischen Praxis gehören zunehmend forensisch-medizinische Untersuchungen von Lebenden. Im Gegensatz zu Studien über Gewaltopfer findet sich in der Fachliteratur allerdings ein erhebliches Defizit an Ergebnissen über systematische rechtsmedizinische Untersuchungen von Tatverdächtigen.

Fragestellung. Welche relevanten Daten können aus einer retrospektiven Analyse von forensisch-medizinischen Untersuchungen von Tatverdächtigen erhoben und welche Rückschlüsse für die rechtsmedizinische Praxis gezogen werden?

Material und Methode. Es wurden insgesamt 270 Gutachten nach forensisch-medizinischen Untersuchungen von Tatverdächtigen aus dem Einzugsgebiet des Instituts für Rechtsmedizin der Universitätsmedizin Rostock der Jahre 2006 bis 2018 nach vorab definierten Kriterien ausgewertet.
Ergebnisse. Die vorgeworfenen Straftaten waren am häufigsten Körperverletzungen ( $n=88=30,7 \%)$, gefolgt von vorsätzlichen Tötungen $(n=63=22,0 \%)$ und Sexualdelikten $(n=49=17,1 \%)$. Von den begutachteten Personen waren $236(87,4 \%)$ männlich und $34(12,6 \%)$ weiblich. Die Mehrzahl der Tatverdächtigen ( $n=175=65,5 \%)$ war in einem Lebensalter von 18 bis 40 Jahren. Im Hinblick auf die Aussage der rechtsmedizinischen Ergebnisse in Bezug auf den vorgeworfenen Straftatbestand wurden $126(46,7 \%)$ Gutachten als für den Tatverdächtigen belastend, 13 (4,8\%) als entlastend und 131 (48,5\%) als frei von Beoder Entlastungsaussagen eingestuft. Zu den 270 Gutachten über Tatverdächtige gab es 209 (77,4\%) korrespondierende Opferuntersuchungen. Von diesem Teilkollektiv mit Täter-Opfer-Begutachtungen wurden in
193 Fällen (92,3 \%) Tatverdächtige und Opfer von demselben Gutachter untersucht. Schlussfolgerungen. Für die Rekonstruktion eines Tatgeschehens sind rechtsmedizinische Untersuchungen des Opfers und des Tatverdächtigen wertvoller als die nicht selten beobachtete Untersuchung der geschädigten Person allein. Befunde mit belastenden Tendenzen für den Tatverdächtigen werden häufiger festgestellt als solche, die zu einer Entlastung des Beschuldigten führen. Da es in der Fachliteratur im Vergleich zu den Opfern von Gewalttaten ein Defizit an Ergebnissen über systematische forensisch-medizinische Untersuchungen von Tatverdächtigen gibt, sollten weitere Studien folgen.

Schlüsselwörter

Kriminalität · Beweis · Verletzungen . Forensische Spuren · Rekonstruktion

\section{Forensic medical examinations of persons suspected of a crime. An analysis of $\mathbf{2 7 0}$ expertises from 2006 to 2018}

\section{Abstract}

Background. Physical examinations of living persons are increasingly part of the forensic medical practice. In contrast to studies on victims of violence, there is a considerable lack of results on systematic physical examinations of persons suspected of a crime in the literature.

Objective. Which relevant data can be observed from a retrospective analysis of physical examinations of persons suspected of a crime and which conclusions can be drawn for forensic medical practice.

Methods. A total of 270 expertises based on physical examinations of persons suspected of a crime from the catchment area of the Institute for Legal Medicine of the Rostock University Medical Center from 2006 to 2018 were evaluated according to prior defined criteria.
Results. The most common criminal offenses were bodily harm $(n=88)$, followed by homicides $(n=63)$ and sexual offenses $(n=49)$. Of the persons examined, 236 were male and 34 were female. Most of the persons suspected of a crime $(n=175,65.5 \%)$ were between 18 and 40 years of age. With regard to the tendency of the results to provide information in relation to the alleged criminal offense, $126(46.7 \%)$ reports were classified as incriminating for the suspect, $13(4.8 \%)$ as exonerating and 131 (48.5\%) as free from incriminating or relief tendencies. Of the 270 reports on suspects, there were 209 (77.4\%) corresponding victim investigations. Of this sub-collective with perpetrator-victim assessments, suspects and victims were examined by the same expert in 193 cases (92.3\%).
Conclusion. For the reconstruction of a crime, physical examinations of the victim and the alleged criminal are more valuable than the often observed examination of the injured person alone. Findings with negative tendencies for the suspect are found more frequently than those which lead to the accused being exonerated. Since there is a deficit in the results of systematic physical examinations of persons suspected of a crime in the literature compared to the victims of violent crimes, further studies should follow.

Keywords Crime $\cdot$ Proof $\cdot$ Injury $\cdot$ Forensic traces . Reconstruction

\section{Beispiel eines Gutachtens mit belastender Aussage}

Kriminalfälle nur ausgewählte Aspekte berücksichtigt werden konnten.

\section{Vorgeschichte}

Im Sommer 2013 wurde eine junge Frau in Sportbekleidung auf dem Grenzweg vorgestellt. Dabei wird um Verständnis 


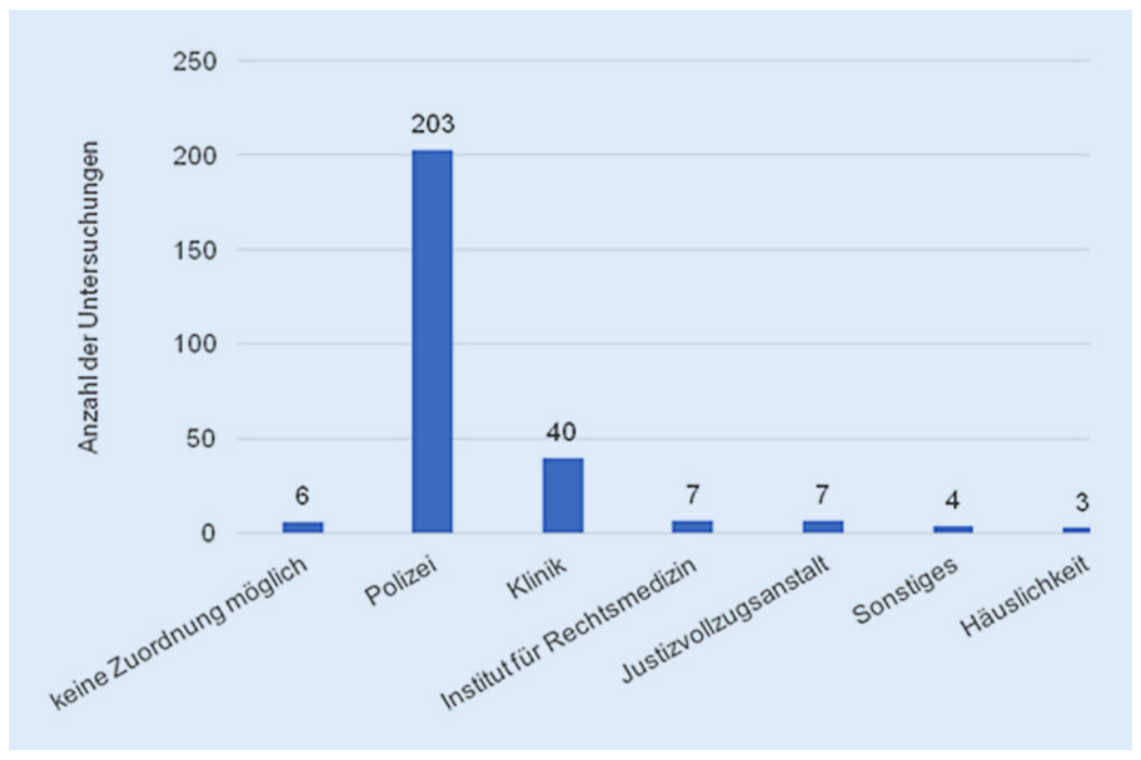

Abb. $3 \triangle$ Absolute Häufigkeit der Untersuchungen nach räumlicher Lokalisation

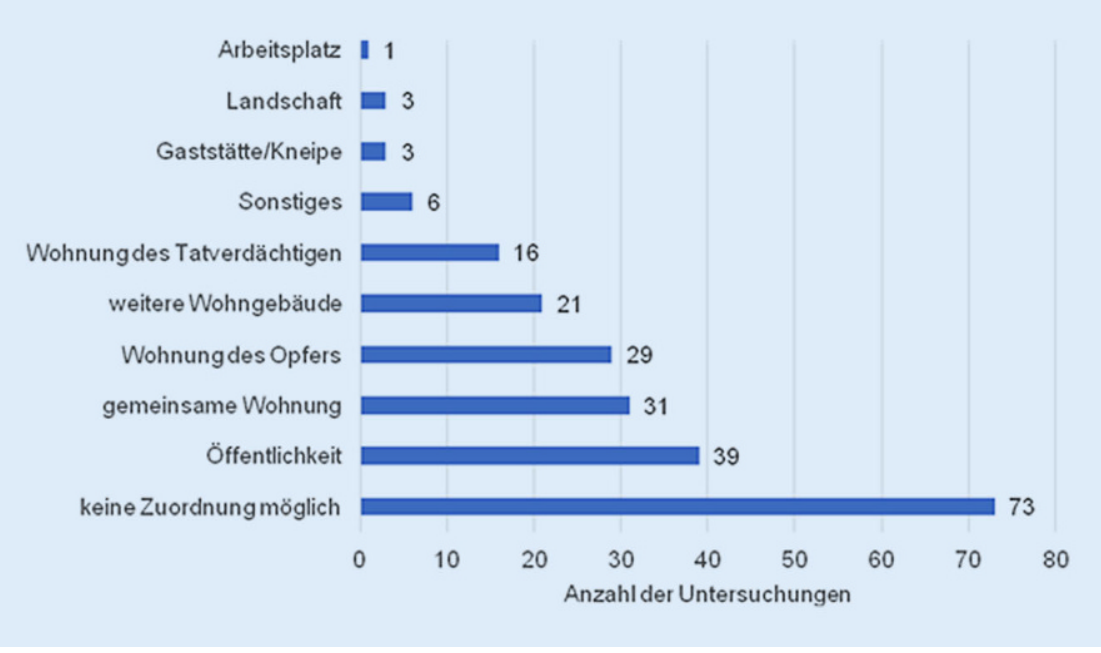

Abb. 4 \ Absolute Häufigkeit der Tatgeschehen nach räumlichem Tatort

zwischen Schleswig-Holstein und Mecklenburg mit einer Halsstichverletzung tot aufgefunden. Am Auffindungsort wurde kein Tatwerkzeug gefunden. Die polizeilichen Ermittlungen ergaben, dass das Opfer zum Joggen aufgebrochen war. Die gerichtliche Sektion erbrachte als Todesursache Verbluten und aktive Abwehrverletzungen an der linken Hand, sodass von einem vorsätzlichen Tötungsdelikt auszugehen war. Am darauffolgenden Tag wurde durch ein spielendes Kind im Beisein der Eltern ein im lockeren Sand verstecktes Butterflymesser, etwa $300 \mathrm{~m}$ vom Auffindungsort entfernt, entdeckt. Eine DNA-Analyse erbrachte die DNA des Opfers und eines polizeibekannten zeit 4 Tage zuvor vereinbar und charakteristisch für eine oberflächliche Schnittverletzung. Nach Kenntnis der Todesursache und der Abwehrverletzungen der Frau sowie des nach dem DNA-Gutachten in Betracht zu ziehenden Tatwerkzeuges Butterflymesser wurde dieser Befund als Belastung für den Tatverdächtigen gewertet.

\section{Ausgang des Strafverfahrens}

In der Gerichtsverhandlung war die Verletzung am Finger des tatverdächtigen Mannes einer von zahlreichen Indizienbeweisen gegen den nichtgeständigen Angeklagten. Die Strafkammer verurteilte den 45-jährigen Mann wegen Mordes zu einer lebenslangen Freiheitsstrafe und stellte weiterhin die besondere Schwere der Schuld fest. In der Urteilsbegründung finden sich die Mordmerkmale „Heimtücke“ und „aus niederen Beweggründen“.

\section{Beispiel eines Gutachtens mit entlastender Aussage}

\section{Vorgeschichte}

Zwei Männer, die sich zum Trinken von Alkohol in einer Wohnung in der 6. Etage eines Mehrfamilienhauses verabredet hatten, gerieten in einen heftigen Streit miteinander. Der 20-jährige Herr B. schlug den 23-jährigen Herrn S. mit einer Holzstrebe eines Stuhls, der daraufhin in die Küche lief und die Tür hinter sich verbarrikadierte. Herr B. schloss nun die Wohnungstür ab, sodass eine Flucht des Herrn S. aus der Wohnung unmöglich war. Daraufhin trat Herr B. die Küchentür ein. Als Herr B. die Küche betrat, lief Herr S. sofort hinaus und stach dabei mit einem Küchenmesser einmal in die Brust des Herrn B., um diesen kampfunfähig zu machen. Der getroffene Herr B. wurde durch den gerufenen Notarzt unter Reanimationsmaßnahmen in eine Klinik gebracht, in der er noch während einer Notoperation verstarb. Die gerichtliche Sektion erbrachte einen Stich des Rumpfes mit Verletzungen der linken Herzkammer und linken Lunge als Todesursache. 


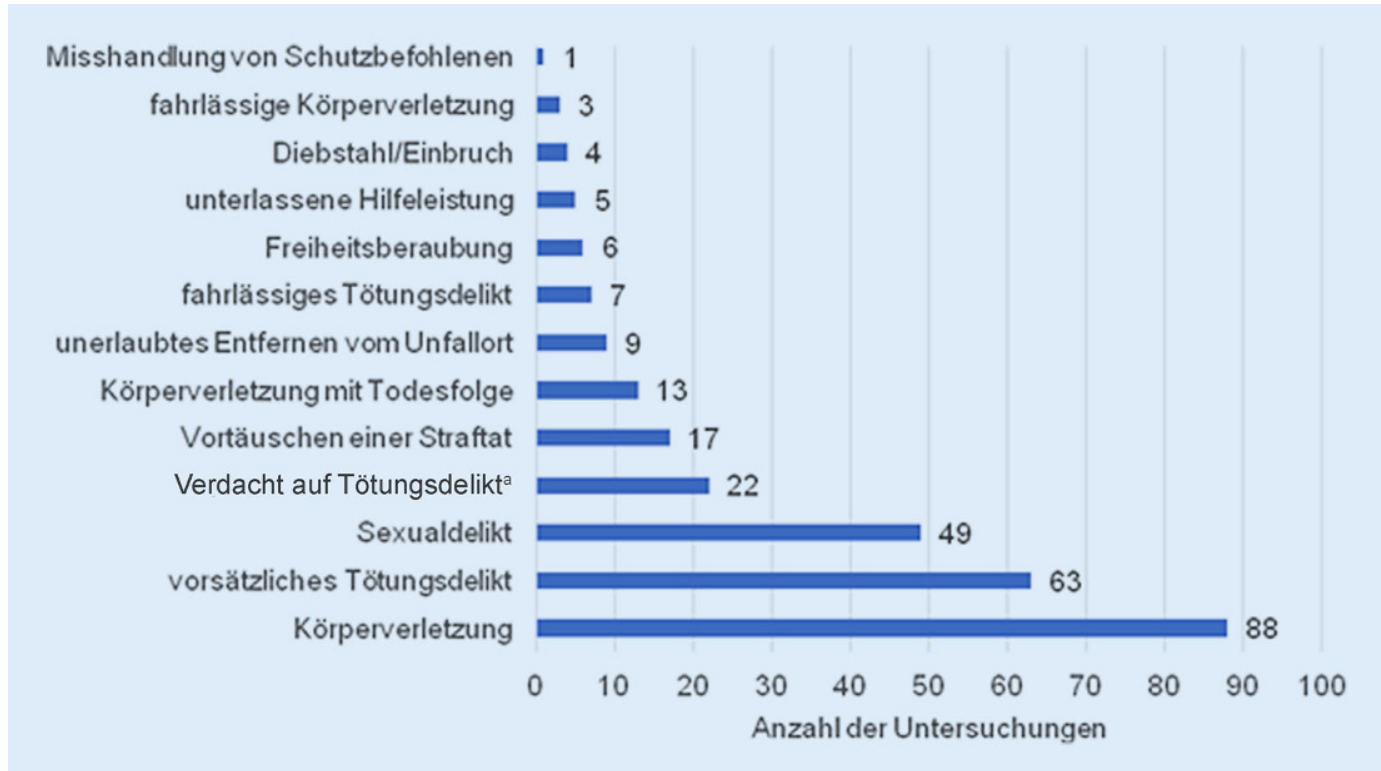

Abb. $5 \triangleleft$ Absolute Häufigkeit der Qualifikation der Tat aus rechtsmedizinischer Sicht ('Verdacht nach polizeilichen Ermittlungen und/oder Obduktionsergebnis nicht bestätigt, Mehrfachzuordnungen für einen Tatverdächtigen möglich)

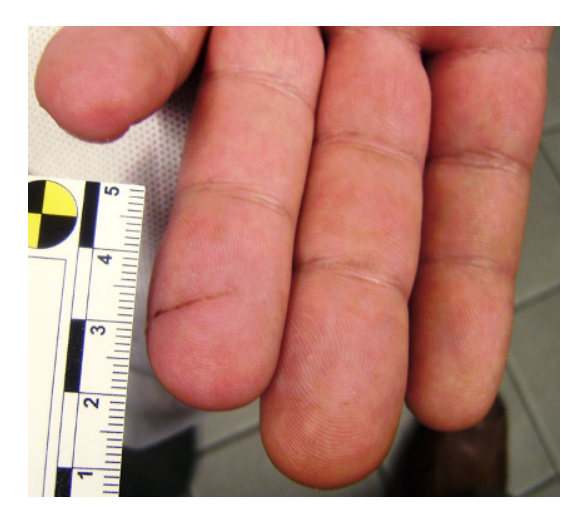

Abb. $6 \Delta$ In Abheilung befindliche glattrandige Verletzung der Innenseite des Endgliedes des Ringfingers der linken Hand (Tatwerkzeug: Butterflymesser)

\section{Rechtsmedizinische Begutachtung}

Die körperliche Untersuchung des Tatverdächtigen $14 \mathrm{~h}$ nach der Tat erbrachte zahlreiche stockschlagtypische Verletzungen des Rumpfes und beider Arme, eine blutverkrustete Hautverletzung der Streckseite des linken Unterarmes und des rechten Handrückens ( $\bullet$ Abb. 7 und 8). Im Rahmen dieser Untersuchung wurde dem Rechtsmediziner das mutmaßliche Schlagwerkzeug, eine Strebe eines Holzstuhles, vorgelegt (• Abb. 9).

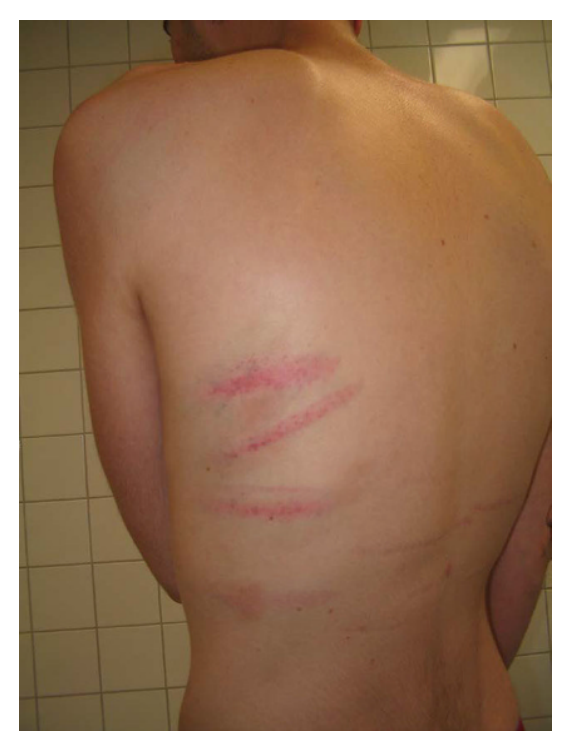

Abb. 7 ^ Zahlreiche stockschlagtypische Hautverletzungen bevorzugt der linken Rumpfseite

\section{Ausgang des Ermittlungsverfah- rens}

Das Ermittlungsverfahren gegen Herrn S. wegen Körperverletzung mit Todesfolge wurde nach Vorliegen der Beschuldigtenvernehmung, Tatortauswertung, Zeugenaussagen und Ergebnisse der rechtsmedizinischen Untersuchungen gemäß $\$ 170$ Abs. 2 StPO durch eine Verfügung des zuständigen Staatsanwaltes eingestellt. Die Tat wurde als eine durch Notwehr gerechtfertigte Handlung angesehen.

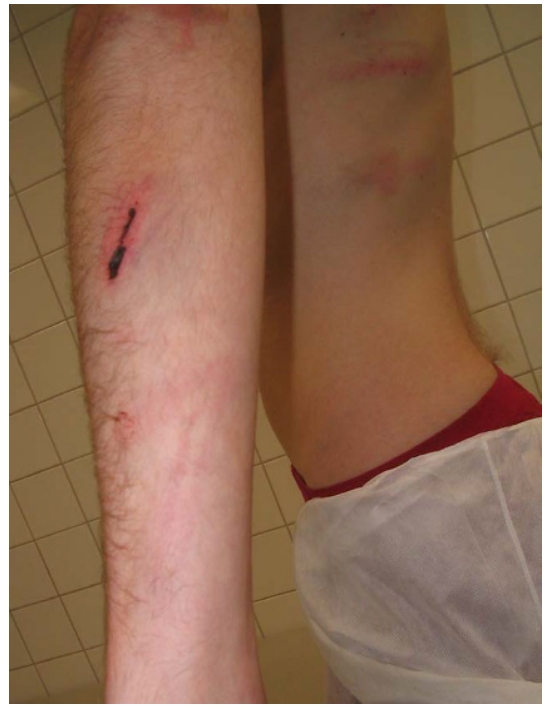

Abb. 8 A Eine längliche blutverkrustete Hautverletzung mit umgebender Rötung an der Streckseite des linken Unterarmes

\section{Beispiel eines Gutachtens ohne be- oder entlastende Aussage}

\section{Vorgeschichte}

Der 18-jährige Herr D. und der 28-jährige Herr L. geraten auf einer Internetplattform über die richtige Lösung einer Mathematikaufgabe, die Herr D. ins Netz gestellt hatte, in einen Streit. Dabei kam es zu wechselseitigen Beleidigungen der Männer, die sich zuvor nicht gekannt hatten. Am nächsten Tag erkannte der gekränkte Herr L., der mit Herrn D. in der- 


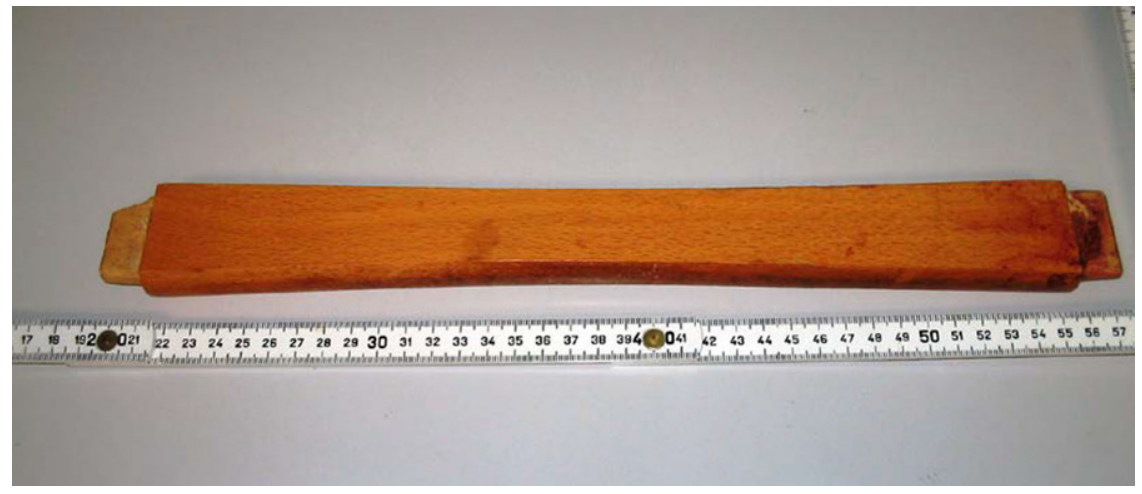

Abb. $9 \Delta$ Die Strebe eines Holzstuhles mit bluttypischen Verschmutzungen am rechten Ende als Schlagwerkzeug

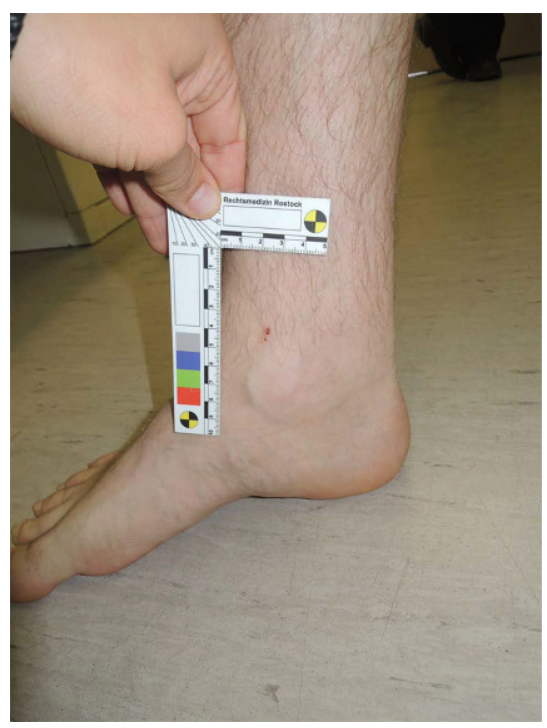

Abb. $10 \Delta$ Diskrete verkrustete Hautverletzung oberhalb des Innenknöchels des rechten Sprunggelenks

selben Stadt lebte, diesen aufgrund des Profilbildes von der Internetplattform auf der Straße. Er stach dem jüngeren Mann im Vorbeigehen ein mitgeführtes Messer in den Bauch. Als der verletzte Mann sich wegdrehte, um wegzulaufen, stach Herr L. dem Herrn D. in den Rücken. Als Herr D. daraufhin zu Boden ging, erfasste Herr L. den Kragen des verletzten Mannes und beschimpfte ihn. Danach verließ er ruhigen Schrittes den Tatort. In einer am selben Tag bei Herrn D. erfolgten Notoperation wurden eine Verletzung der Magenwand und der A. renalis dextra festgestellt und versorgt. Der Mann, der viel Blut verloren hatte, überlebte die Verletzungen, verlor jedoch aufgrund einer irreversiblen Mangeldurchblutung seine rechte Niere.

\section{Rechtsmedizinische Begutachtung}

Diekörperliche Untersuchung des $192 \mathrm{~cm}$ großen Tatverdächtigen etwa $20 \mathrm{~h}$ nach der Tat erbrachte neben einer uncharakteristischen diskreten Hautläsion oberhalb des Innenknöchels des rechten Sprunggelenks (•Abb. 10) keine weiteren Verletzungen oder auffällige Befunde.

\section{Ausgang des Strafverfahrens}

Das Landgericht verurteilte den geständigen Herrn L., der zum Zeitpunkt der Verhandlung bereits $20.000 €$ Schmerzensgeld an Herrn D. gezahlt hatte, wegen des versuchten Totschlags zu einer Freiheitsstrafe von 5 Jahren und 9 Monaten. Aus dem Gutachten über die körperliche Untersuchung des Tatverdächtigen wurde im Urteil lediglich die Körpergröße, die ähnlich wie die des Herrn D. war, erwähnt.

\section{Diskussion}

Das Ziel einer körperlichen Untersuchung eines Tatverdächtigen ist nach den Kommentaren zum $\$$ 81a StPO die Feststellung verfahrenserheblicher Tatsachen [9]. Da diese fallabhängig erheblich differieren können, ist eine Konkretisierung der Fragestellung/en für den Gutachter empfehlenswert, mitunter sogar erforderlich. Bei lediglich $21 \%$ der beauftragten Untersuchungen von Tatverdächtigen dieser Studie wurden vom Veranlasser zum Zeitpunkt der Auftragserteilung Fragen an den Gutachter gestellt. Vergleichbare Ergeb- nisse fanden sich, bevorzugt aufgrund eines Defizits an Studien, im Schrifttum nicht. Gleiches gilt für die Häufigkeit einer Spurensicherung im Rahmen einer Untersuchung von Beschuldigten. Sobald das Ziel oder der Umfang einer Untersuchung problematisch sein sollte, empfiehlt sich eine rechtzeitige Rücksprache mit dem Auftraggeber.

Da sich naturgemäßzahlreiche Befunde sowohl beim Tatverdächtigen als auch beim Opfer im Laufe der Zeit verändern bzw. nicht mehr nachweisbar sein können, ist eine kurze Differenz zwischen Tatzeit und Untersuchung anzustreben. Eine Untersuchungsrate von $60,7 \%$ innerhalb der ersten $24 \mathrm{~h}$ nach der Tat spiegelt wider, dass dieser in der Rechtsmedizin anerkannte Grundsatz bei der Mehrzahl der Gutachten dieser Studie berücksichtigt wurde [10]. Ein mit 56,6\% ähnliches Ergebnis wurde in einer vergleichbaren Hamburger Studie über 99 Untersuchungen von Tatverdächtigen erreicht [7]. Es kann jedoch nicht jeder Fall innerhalb der ersten Stunden nach der Tat untersucht werden, da es z. B. auch verspätete Anzeigen bei der Polizei oder Ergreifungen von Tatverdächtigen nach tagelangen Ermittlungen gibt. Gegebenenfalls kann es für die Befundbewertung hilfreich sein, den Beschuldigten nach einer Latenz von z.B. $24 \mathrm{~h}$ ein zweites Mal zu begutachten [7].

Da sich die Mehrzahl der Beschuldigten zur Untersuchungszeit in Polizeigewahrsam befand, ist das Ergebnis, dass drei von vier Tatverdächtigen in Räumen der Polizei untersucht worden waren, naheliegend.

Zur Vermeidung von Informationsverlusten ist anzustreben, dass Opfer und Tatverdächtiger in einem Strafverfahren von demselben Sachverständigen untersucht werden [10]. In 92,3\% aller Fälle mit Opfer- und Täteruntersuchungen wurde dieser Grundsatz in der vorliegenden Studie weitestgehend berücksichtigt. Der Grund, dass es bei den 270 Untersuchungen von Beschuldigten in 61 Fällen keine Opferuntersuchung gab, liegt auch in der Zusammenstellung des Studienkollektivs. So waren z. B. bei Untersuchungen von Tatverdächtigen, denen ein Einbruch oder eine Vortäuschung einer 
Straftat vorgeworfen wurde, in der Regel keine Opfer zu begutachten.

Mit 71,9\% wurde die Mehrzahl der Taten dieser Studie von alleinhandelnden Beschuldigten verübt. In der Polizeilichen Kriminalitätsstatistik (PKS) für Deutschland 2019 ist dieser Anteil mit $82,6 \%$ noch höher [11]. Ein Grund dafür liegt offensichtlich in der Zusammensetzung der Fallgruppen, denn körperliche Untersuchungen der Beschuldigten sind bei Straftaten wie z.B. Betrug oder Beleidigung nicht indiziert.

Dass bei den Tatorten die Öffentlichkeit vor der Wohnung des Opfers dominierte, zeigte sich auch bei vorherigen Studien desselben Einzugsgebietes, bei denen hingegen nur vorsätzliche Fremdtötungen ausgewertet wurden $[12,13]$. Die unerwartet große Anzahl an fehlenden Angaben zum Tatort in den Gutachten führte zu einer institutsinternen Auswertung.

Die den Tatverdächtigen vorgeworfenen Straftaten waren am häufigsten vorsätzliche Körperverletzungen, gefolgt von vorsätzlichen Tötungen und Sexualdelikten im Verhältnis 1,4:1:0,8. Betrachtet man diese ausgewählten Straftatengruppen in der aktuellen PKS für Deutschland 2019, so ergibt sich ein Verhältnis von Körperverletzungen (vorsätzliche einfache, gefährliche und schwere): vorsätzlichen Tötungen (Mord, Totschlag und Tötung auf Verlangen): Straftaten gegen die sexuelle Selbstbestimmung (Vergewaltigung, sexuelle Nötigung und sexueller Übergriff) von 154:1:2,7 [11]. Demnach kam es in Bezug auf die Gesamtheit einer Tatgruppe im Einzugsgebiet des Rostocker Instituts am häufigsten bei vorsätzlichen Tötungsdelikten zu körperlichen Untersuchungen der Beschuldigten nach $\$ 81$ a StPO.

Sowohl in der Kriminalitätsstatistik als auch in der Fachliteratur dominieren bei der Aufteilung nach dem Geschlecht die männlichen Tatverdächtigen erheblich [7, 11-13]. In der aktuellen PKS für Deutschland betrug der Anteil des männlichen Geschlechts an den gesamten Straftaten 2019 75,0\%, in der vorliegenden Studie 87,4\% [11]. Ein Grund für den höheren Anteil des männlichen Geschlechts in der Rostocker Studie liegt unseres Erachtens an dem hohen An- teil gewaltbedingter Straftaten im Zusammenhang mit der Beauftragung der Untersuchungen nach $\$ 81$ a StPO. In einer Hamburger Studie mit 35 Begutachtungen von Beschuldigten, denen vorwiegend versuchte oder vollendete Fremdtötung vorgeworfen worden war, betrug der männliche Anteil sogar 94,3\% [7].

Dass die Mehrzahl der Tatverdächtigen der Studie in einem Lebensalter von 18 bis 40 Jahren war, spiegelt sich auch in der PKS für die gesamte Bundesrepublik wider [11].

Von 231 untersuchten Personen mit bekannter Nationalität waren 19 (8,2\%) Nichtdeutsche. In der PKS für die Bundesrepublik 2019 war der Anteil der Nichtdeutschen unter 2.019.211 Tatverdächtigen mit 699.261 (34,6\%) Personen deutlich höher. Ein Grund dafür dürfte der geringere Anteil an Nichtdeutschen an der Bevölkerung in MecklenburgVorpommern (5,0\%) im Vergleich zum Anteil der ausländischen Bevölkerung an der Gesamtpopulation in Deutschland $(13,5 \%)$ sein [14-16].

In dieser Studie hatte jedes zweite Gutachten in der Zusammenfassung eine entweder be- oder entlastende Aussage für den Tatverdächtigen in Bezug auf den Tatvorwurf. Das Verhältnis von 126:13 Gutachten zugunsten der belastenden Aussage zeigte eine 9-mal höhere Wahrscheinlichkeit dafür, dass die angeordneten Untersuchungen Befunde mit Belastung für den Tatverdächtigen erbrachten, jedoch von vornherein keine entlastenden Aspekte hinsichtlich des Tatvorwurfs dokumentiert wurden. Der Anteil der Gutachten mit belastender Aussage (46,7\%) lag etwas höher als in der Hamburger Studie (35,5\%) [7]. Bei der Komplexität der Thematik wäre die Suche nach einer Ursache für diese Differenz eher spekulativ.

Die wenigen vorliegenden Übersichten in der Fachliteratur über rechtsmedizinische körperliche Untersuchungen von Tatverdächtigen betreffen bevorzugt Verletzungen durch scharfe Gewalt im Rahmen von Messerattacken. Die Autoren beschreiben, wie in 0 Abb. 6 dieser Studie beispielhaft dargestellt, glattrandige Verletzungen der Hände der Tatverdächtigen [8, 17, 18]. Diese Verletzungen sind oberflächlich und bevor- zugt an der Beugeseite der Hände lokalisiert. Die dokumentierten Befunde lassen sich durch ein Abrutschen der Hand auf die Klinge beim Zustechen mit dem Messer erklären. Aber auch bei anderen Gewaltstraftaten wie z. B. Sexualdelikten, Schusswaffenanwendung, Faustkämpfen oder zur Klärung der Sitzpositionen von Fahrzeuginsassen nach Verkehrsdelikten können die Untersuchungen der Tatverdächtigen wertvolle Befunde für den Beweis der Täterschaft oder deren Widerlegung erbringen [10, 19-21].

\section{Fazit für die Praxis}

- Für die Rekonstruktion eines Tatgeschehens sind rechtsmedizinische Untersuchungen des Opfers und des Tatverdächtigen wertvoller als die nicht selten beobachtete alleinige Untersuchung der geschädigten Person.

- Befunde mit belastenden Aussagen für den Tatverdächtigen werden häufiger festgestellt als solche, die zu einer Entlastung des Beschuldigten führen.

- Befunde mit entlastenden Aussagen können bei einem Tötungsdelikt eine Notwehrsituation belegen.

- Da es in der Fachliteratur im Vergleich zu den Opfern von Gewalttaten ein Defizit an Ergebnissen über systematische forensisch-medizinische Untersuchungen von Tatverdächtigen gibt, sollten weitere Studien folgen, ggf. mit dem Fokus auf einen ausgewählten Straftatbestand oder einer definierten traumatologischen Einwirkung.

\section{Korrespondenzadresse}

Prof. Dr. med. F. Zack

Institut für Rechtsmedizin, Universitätsmedizin Rostock

St.-Georg-Str. 108, 18055 Rostock, Deutschland fred.zack@med.uni-rostock.de

Funding. Open Access funding enabled and organized by Projekt DEAL. 


\section{Einhaltung ethischer Richtlinien}

Interessenkonflikt. F. Zack, S. Bernhardt, C. Arnold und A. Büttner geben an, dass kein Interessenkonflikt besteht.

Alle beschriebenen Auswertungen von Untersuchungen am Menschen wurden mit Zustimmung der zuständigen Ethikkommission (Antragsnummer: A 2018-0215), im Einklang mit nationalem Recht sowie gemäß der Deklaration von Helsinki von 1975 (in der aktuellen, überarbeiteten Fassung) durchgeführt.

Open Access. Dieser Artikel wird unter der Creative Commons Namensnennung 4.0 International Lizenz veröffentlicht, welche die Nutzung, Vervielfältigung, Bearbeitung, Verbreitung und Wiedergabe in jeglichem Medium und Format erlaubt, sofern Sie den/die ursprünglichen Autor(en) und die Quelle ordnungsgemäß nennen, einen Link zur Creative Commons Lizenz beifügen und angeben, ob Änderungen vorgenommen wurden.

Die in diesem Artikel enthaltenen Bilder und sonstiges Drittmaterial unterliegen ebenfalls der genannten Creative Commons Lizenz, sofern sich aus der Abbildungslegende nichts anderes ergibt. Sofern das betreffende Material nicht unter der genannten Creative Commons Lizenz steht und die betreffende Handlung nicht nach gesetzlichen Vorschriften erlaubt ist, ist für die oben aufgeführten Weiterverwendungen des Materials die Einwilligung des jeweiligen Rechteinhabers einzuholen.

Weitere Details zur Lizenz entnehmen Sie bitte der Lizenzinformation auf http://creativecommons.org/ licenses/by/4.0/deed.de.

\section{Literatur}

1. Banaschak S, Gerlach K, Seifert D, Bockholdt B, Graß H (2014) Forensisch-medizinische Untersuchung von Gewaltopfern. Rechtsmedizin 24:405-411

2. Brackrock D, Dokter M, Eckhoff C, Philipp KP, Bockholdt B (2020) Gewalt gegen Frauen - eine Auswertung von sieben Jahren Gewaltopferambulanz am Institut für Rechtsmedizin Greifswald (2011-2017). Rechtsmedizin 30:153-160

3. Kolbe V, Büttner A (2020) Häusliche Gewalt gegen Männer - auch ein rechtsmedizinisches Problem? Rechtsmedizin 30:88-93

4. Mützel E, Helmreich C, Schick S, Saß M, Schöpfer J (2014) Klinisch-forensische Versorgung von Gewaltopfern in Bayern. Rechtsmedizin 24:200-207

5. Todt M, Awe M, Roesler B, Germerott T, Debertin AS, Fieguth A (2016) Häusliche Gewalt. Daten, Fakten und Herausforderungen. Rechtsmedizin 26:499-506

6. Zinka B, Schick S, Mützel E (2019) Auswertung der Untersuchungsdaten der Bayerischen Kinderschutzambulanz des Instituts für Rechtsmedizin der Universität München aus den Jahren 2014-2017. Rechtsmedizin 29:13-20

7. Naeve W, Lohmann E (1973) Methodik und Beweiswert körperlicher Sofort-Untersuchungen lebender Personen nach Straftaten. Z Rechtsmed 72:79-99

8. Schmidt U, Pollak S (2006) Sharp force injuries in clinical forensic medicine - findings in victims and perpetrators. Forensic Sci Int 159:113-118
9. Meyer-Goßner L, Schmitt B (2020) Strafprozessordnung mit Gerichtsverfassungsgesetz und Nebengesetzen, 63. Aufl. Beck, München

10. Grassberger M, Türk EE (2013) Die körperliche Untersuchung von Tatverdächtigen im Rahmen des Strafverfahrens. In: Grassberger M, Türk EE, Yen K (Hrsg) Klinisch-forensische Medizin. Springer, Wien New York, S119-126

11. Bundeskriminalamt (2020) Polizeiliche Kriminalitätsstatistik Bundesrepublik Deutschland Jahrbuch 2019 Band 3 Tatverdächtige. https://www.bka.de/DE/Aktuellelnformationen/ StatistikenLagebilder/PolizeilicheKriminalstatistik/ PKS2019/PKSJahrbuch/pksJahrbuch node.html. Zugegriffen: 18. Jan. 2021

12. George C (2006) Synopsis der Tötungsdelikte im Landgerichtsbezirk Rostock 1992-2001 unter Berücksichtigung kriminologischer Aspekte. Med Diss. Universität Rostock, Rostock

13. Leder JC (2004) Synopsis der Tötungsdelikte im Landgerichtsbezirk Schwerin (1992-2001) unter besonderer Berücksichtigung des kriminogenen Faktors Alkohol. Med Diss. Universität Rostock, Rostock

14. Landesamt für innere Verwaltung Statistisches Amt Mecklenburg-Vorpommern (2020) Bevölkerung insgesamt und ausländische Bevölkerung seit 1991. https://www.laiv-mv.de/static/LAIV/ Statistik/Dateien/Publikationen/A\%20I\%20Bev \%C3\%B6lkerungsstand/A143/A143\%202019 \%2000.pdf.Zugegriffen: 19.Jan. 2021

15. Statistisches Bundesamt (2020) Ausländische Bevölkerung nach Geschlecht und ausgewählten Staatsangehörigkeiten. https://www. destatis.de/DE/Themen/Gesellschaft-Umwelt/ Bevoelkerung/Migration-Integration/Tabellen/ auslaendische-bevoelkerung-geschlecht.html jsessionid =C5D54AE3940DFB5185171CF3AD 330A50.internet8722.Zugegriffen: 19. Jan. 2021

16. Statistisches Bundesamt (2020) Bevölkerungsstand. https://www.destatis.de/DE/ Themen/Gesellschaft-Umwelt/Bevoelkerung/ Bevoelkerungsstand/Tabellen/zensus-geschlechtstaatsangehoerigkeit-2020.html. Zugegriffen: 19. Jan. 2021

17. Schmidt U, Faller-Marquardt $M$, Tatschner $T$ Walter K, PollakS (2004) Cuts to the offender's own hand-unintentional self-infliction in the course of knife attacks. Int J Legal Med 118:348-354

18. Tsokos M, Braun C (2005) Das Verletzungsbild an der Hand durch Abrutschen auf die Klinge beim Zustechen mit einem Messer. Arch Krimino 215:1-10

19. Pollak S (2015) Schussverletzungen. In: Madea (Hrsg) Rechtsmedizin, 3. Aufl. Springer, Berlin Heidelberg, S245-262

20. van Oorschot RAH, Szkuta B, Meakin GE, Kokshoorn B, Goray M (2019) DNA transfer in forensic science:a review. Forensic ScilntGenet38:140-166

21. Kleemann WJ, Schroeder G, Eidam J, Urban R, Tröger HD (1993) Forensisch-klinische Untersuchung LebenderzurVerkehrsunfallrekonstruktion. Rechtsmedizin 4:26-31
Auch online Zugang zu allen Beiträgen Ihres Zeitschriftenabonnements

Wussten Sie, dass Sie als Abonnent*in dieser Zeitschrift automatisch Online-Zugriffsrechte auf das gesamte Beitragsarchiv und die CMEKurse haben?

Der Zugang zu Ihrer Online-Bibliothek und den CME-Kursen ist im Abonnement Ihrer Zeitschrift inbegriffen. Für den Zugang müssen Sie sich lediglich einmal über www.springermedizin.de/register registrieren.

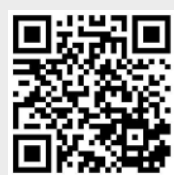

Über diesen QR-Code schnell und einfach registrieren

Bitte nutzen Sie für die Registrierung Vorund Nachname und Lieferadresse wie beim Abonnement der Zeitschrift (s. Adressaufkleber auf Ihrem Heft). So kann im System die Zugehörigkeit zu Ihrer Zeitschrift sichergestellt werden.

Aufgrund des Heilmittelwerbegesetzes dürfen die Inhalte der Website nur medizinischen Fachkreisen zur Verfügung gestellt werden. Bei der Anmeldung bitten wir Sie deshalb, einen Berufsnachweis vorzulegen. Bei einer Mitgliedschaft in der deutschen Ärztekammer reicht die Angabe der einheitliche Fortbildungsnummer (EFN). Alternativ schicken Sie eine Bestätigung des Arbeitgebers, Studiennachweis mit Angabe des Studiengangs ganz unkompliziert an kundenservice@springermedizin.de.

Mit Benutzername und Passwort haben Sie außerdem Zugang zu den freien Inhalten auf den Seiten von: www.springermedizin.de www.aerztezeitung.de

Sollten Fragen oder Probleme auftauchen, wenden Sie sich einfach an Ihren Kundenservice: kundenservice@springermedizin.de 